\title{
Coherent Extreme Ultraviolet Light Amplification by Strong-Field-Enhanced Forward Scattering
}

\author{
Carles Serrat* \\ UPC-Universitat Politècnica de Catalunya, Colom 11, 08222 Terrassa (Barcelona), Spain
}

(Received 12 June 2013; published 24 September 2013)

\begin{abstract}
We theoretically study the response of He atoms exposed simultaneously to an intense IR pulse and a weak extreme ultraviolet (XUV) pulse with photon energies far from the principal atomic He resonances. We find that XUV forward scattering from the nonstationary electronic wave packet promoted by the intense IR driving field is strongly enhanced as compared with the normal weak scattering from bound or free electrons. Based on this effect, we predict that large amplification of XUV radiation can be achieved in the cutoff spectral region of high-harmonic generation in He gas.
\end{abstract}

DOI: 10.1103/PhysRevLett.111.133902

PACS numbers: $42.65 . \mathrm{Ky}, 32.80 . \mathrm{Fb}, 42.55 . \mathrm{Vc}$

The availability of tabletop coherent extreme ultraviolet (XUV) radiation sources with high photon energies has been largely pursued over the last two decades [1]. In this Letter we consider the amplification of the XUV yield generated in high-harmonic generation (HHG) processes by considering the simultaneous excitation of $\mathrm{He}$ atoms with an intense IR driving pulse and an XUV pulse. It is known that synchronization of one or several low-order harmonics of high peak intensities with the driving IR laser pulse can produce a large yield enhancement $[2,3]$. The target medium is in this case strongly ionized due to the XUV photon energies that are considered and due to their high intensity, so that the influence of the combined fields on the HHG spectrum is at spectral regions of higher photon energies than the added XUV pulse. The present research is crucially different from these previous approaches in that we consider weak intensity XUV pulses with photon energies that are far from the principal atomic resonances, with negligible XUV ionization [4]. In our approach, the only affect on the HHG spectrum is at about the same spectral region as the added XUV pulse. Specifically, we address the influence of the high-intensity IR driving pulse on the scattering of XUV radiation from $\mathrm{He}$ atoms in $\mathrm{HHG}$ processes. We find that forward scattering is largely enhanced when an XUV pulse is synchronized with the amplitude maxima of the IR laser pulse. It is shown numerically that this effect can be used for the amplification of coherent XUV radiation in the HHG cutoff spectral region to power levels that might be useful for most applications.

We resolve the interaction of a $\mathrm{He}$ atom with the combined IR laser and XUV pulse by numerically solving the time-dependent Schrödinger equation in the singleatom strong-field approximation [5]. The strong-field approximation describes the interaction with laser light of arbitrary frequency content, accounts for most important quantum effects-such as quantum diffusion of wave packets and quantum interferences, and allows our study to be computationally efficient. The present results apply for low-pressure HHG processes, propagation effects in a higher-pressure gas will be discussed in a future paper. Resonant absorption from $\mathrm{He}$ atoms will be evaluated in the last part of the study.

In the following we consider a 5 fs (FWHM) laser pulse with Gaussian temporal profile, carrier-envelope phase $0^{\circ}$, central wavelength of $800 \mathrm{~nm}$, and peak intensity of $5 \times$ $10^{14} \mathrm{~W} / \mathrm{cm}^{2}$, which interacts with $\mathrm{He}$ (ionization potential $24.59 \mathrm{eV}$ ), producing high-order harmonics with a photon energy cutoff at $\approx 127 \mathrm{eV}$. We compare the harmonics yield generated by the IR pulse alone with the yield produced when a weak XUV field is combined with the IR pulse at different delay times with respect to the IR pulse center. In the results shown in Fig. 1, the XUV field is a Gaussian pulse of 200 as (FWHM), with a central wavelength that corresponds to the 57th harmonic of the fundamental $800 \mathrm{~nm}$ wavelength in Figs. 1(a)-1(c), and to the 87th harmonic in Fig. 1(d). The harmonic yield enhancement is defined as the ratio between the HHG energy yield of a particular harmonic obtained with and without the XUV pulse. Explicitly, the enhancement calculated in Fig. 1 is given by $\int_{H_{-}}^{H_{+}} E_{\mathrm{IR}+\mathrm{XUV}}^{2} d H / \int_{H_{-}}^{H_{+}} E_{\mathrm{IR}}^{2} d H$, where $H_{-}$and $H_{+}$are the photon energies of the previous and next harmonics, respectively, with respect to the harmonic that coincides with the central wavelength of the XUV pulse, so that the integral comprises the bandwidth of a single harmonic. $E_{\mathrm{IR}+\mathrm{XUV}}$ is the HHG spectral field amplitude in the case where the interaction is performed by combining the IR and the XUV pulses, and $E_{\mathrm{IR}}$ corresponds to the case with the IR pulse alone. $d H$ denotes the integration over the photon energy.

The peak intensity of the XUV pulse is defined by the parameter $\alpha=E_{0}^{2} / E_{H}^{2}$, with $E_{0}$ and $E_{H}$ being the amplitudes of the IR and XUV fields, respectively. In Fig. 1(a) the 57th harmonic-well in the HHG plateau region-is considered. In this case $\alpha=10^{14}$, and therefore the peak intensity of the XUV pulse is only $5 \mathrm{~W} / \mathrm{cm}^{2}$. We can observe from Fig. 1(a) that the HHG yield is strongly influenced by the addition of the XUV pulse, and that 

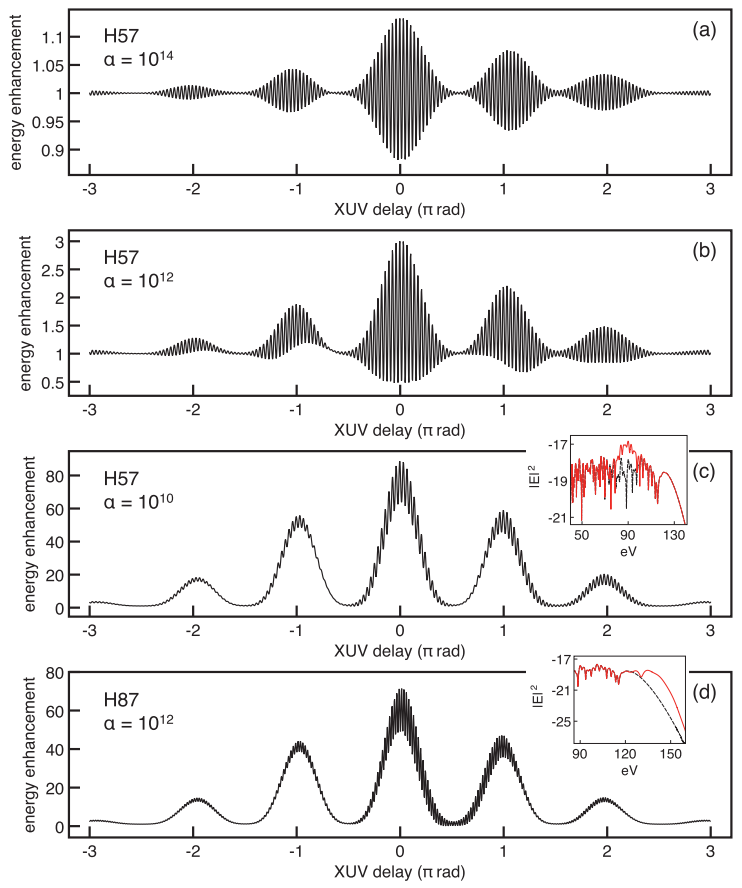

FIG. 1 (color online). Energy enhancement of the 57th $(88.3 \mathrm{eV})$ (a)-(c) and 87 th $(134.8 \mathrm{eV})$ (d) harmonic of the $800 \mathrm{~nm}$ fundamental wavelength, for different ratios between the IR and XUV peak intensities $(\alpha)$, as indicated, as a function of the delay between the IR and the XUV pulse. The delay is given in parts of half a cycle of the IR pulse, in radiants, so that $2 \pi \mathrm{rad} \equiv \lambda_{\mathrm{IR}} / c \approx 2.66 \mathrm{fs}$. The insets in (c) and (d) show the spectra around the 57th and 87th harmonics, respectively, in the case of considering only the IR pulse (black dotted line), and for the IR + XUV pulses (red full line). The vertical axis of the insets is given in logarithmic scale.

this influence is governed by the delay at which the XUV pulse is added. We see that the effect is more important for delays coinciding with higher values of the IR field, in such a way that the shape of the IR pulse is clearly reproduced. Also clear in Fig. 1(a) are the interferences between the XUV field and the electron wave packet that is promoted by the IR field, which produce oscillations on the observed yield enhancement with a period corresponding to the XUV pulse cycle. Figures 1(b) and 1(c) show the calculations performed for increasing values of the XUV peak intensity, with $\alpha=10^{12}$ and $\alpha=10^{10}$, respectively. As the intensity of the XUV pulse increases, its affect on the yield also increases. For $\alpha=10^{10}$, which is shown in Fig. 1(c), the XUV field already dominates, such that the 57th harmonic is enhanced for all delays except where the IR field is null. Figure 1(d) shows the calculations performed by considering the 87th harmonic, which is slightly above the HHG spectrum cutoff, for a value of $\alpha=10^{12}$. The result is qualitatively similar to that of the 57th harmonic in Fig. 1(c), but the enhancement obtained with the 87 th harmonic is much higher, so that with $\alpha=10^{12}$ it already applies to all regions where the IR field is not null.
In what follows we will refer to threshold as the value of $\alpha$ below which the enhancement covers all delays except where the IR field is null, and we will further comment on it later in the Letter. The insets in Figs. 1(c) and 1(d) show the spectra calculated with and without XUV for some particular delays. In Fig. 1(c) the inset shows the results for a delay of $0.206 \pi$, and in Fig. 1(d) the delay is $0.014 \pi$. These spectra show us two important characteristics of the IR + XUV interaction: first, the effect of the XUV pulse on the spectra is only around the XUV central frequency, and, second, the influence of the XUV pulse is observed in a bandwidth that is larger than the XUV bandwidth, which for a transform limited 200 as pulse is $\approx 9 \mathrm{eV}$. In the case of Fig. 1(c) the influence of the XUV pulse on the spectrum covers approximately from 79 to $102 \mathrm{eV}$, while in the case of Fig. 1(d) it starts at $120 \mathrm{eV}$ and includes all the cutoff region.

In order to identify the origin of the observed IR fielddependent enhancement, we have performed a frequencytime analysis (FTA) of the harmonics spectra for some particular cases, and this is shown in Fig. 2. Figure 2(a) shows the FTA of the spectrum obtained with only the IR pulse. Figures 2(b)-2(f) show the FTA considering the addition of the XUV pulse with a central frequency as the 87th harmonic (H87 XUV) in Figs. 2(b) and 2(c), and with that of the 57th harmonic (H57 XUV) in Figs. 2(d)-2(f). The value of $\alpha$ is $10^{12}$ in all cases, and each plot considers a particular delay. Figure 1(d) shows a maximum enhancement when the H87 XUV pulse is added at a delay of $0.014 \pi$ (the delay is given as a fraction of half the $2 \pi$ IR full cycle, in radiants). The FTA for this case is shown in Fig. 2(b). Comparing this result with that of Fig. 2(a), we clearly see a new contribution in the cutoff region, with an
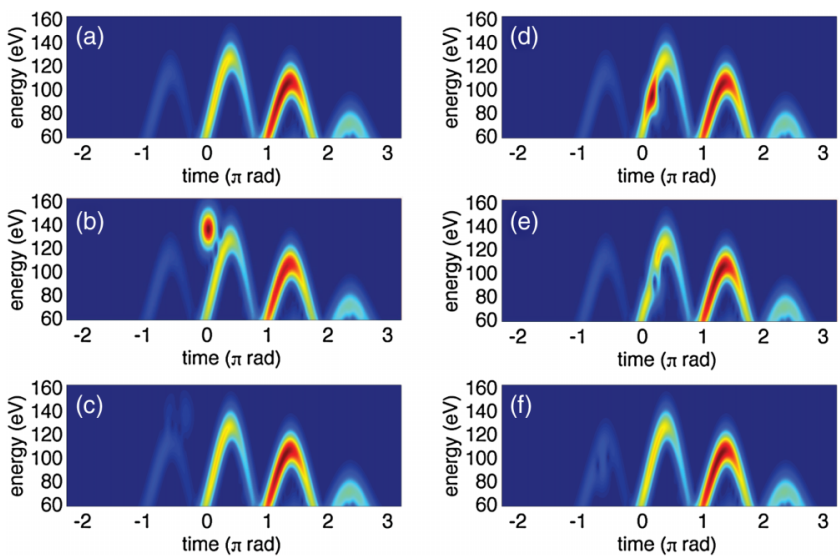

FIG. 2 (color online). FTA of some of the spectra calculated in Fig. 1 with $\alpha=10^{12}$. In (a) the He atom interacts only with the Gaussian IR pulse. In (b) and (c) the IR pulse is combined with its 87 th harmonic at a delay of $0.014 \pi \mathrm{rad}$ and $-0.48 \pi \mathrm{rad}$, respectively. In (d)-(f) the IR pulse is combined with its 57 th harmonic at the delays $0.206 \pi \mathrm{rad}, 0.223 \pi \mathrm{rad}$, and $-0.6 \pi$, respectively. 
intensity maximum at about $135 \mathrm{eV}$, which corresponds to the central photon energy of the 87th harmonic. Most importantly, this contribution appears at the same time (delay) where the XUV pulse has been added, a fact that indicates that this contribution to the total radiation is emitted by forward scattering of the XUV field from the electron wave function, differently from the rest of the radiation that has been emitted by the regular HHG process. Figure 2(c) shows that when the same XUV pulse is added with a delay of $-0.483 \pi$, which corresponds to the region where the IR field is null, no contribution of the XUV radiation is observed. This was already clear from Fig. 1(d) and is now confirmed by the FTA. Figures 2(d)-2(f) correspond to the addition of the H57 XUV pulse, which has a central photon energy of $88.3 \mathrm{eV}$. In this case, the delays that correspond to the IR field maxima basically coincide with the recombination times of the electrons that carry a similar photon energy as the 57th harmonic and that have been tunnel ionized by the IR field. The FTA shows in this case the interference between the scattered XUV pulse and the harmonics emitted by tunneling and recombination. Indeed, in Fig. 2(d) the H57 XUV pulse is added at a delay of $0.206 \pi$, and we can see a clear constructive interference, while a destructive interference is obtained when the XUV pulse is added at a delay of $0.223 \pi$, as shown in Fig. 2(e). In Fig. 2(f) the delay is $-0.6 \pi$, corresponding to the region where the IR field is null, and again the FTA confirms that there is no influence of the XUV pulse on the harmonics spectrum. From the study above we can conclude that the enhancement effect observed in the harmonics spectrum is due to XUV forward scattering from the nonstationary electronic wave packet promoted by the intense IR driving field, which dominates as compared with the normal weak scattering from bound or stationary free electrons. Forward scattering by the nonstationary electron wave function may include several processes between virtual states, such as Raman scattering, high-order parametric processes, or stimulated bremsstrahlung, with a relative contribution that highly depends on the laser parameters and the particular conditions [6].

Let us now examine an application of the observed effect. Can the naturally weak XUV radiation generated in HHG processes be iteratively added to the IR driving pulses, so that an optimal amplification of the XUV radiation is achieved? A simplified scheme of the proposed experimental setup is shown in Fig. 3. A strong IR laser pulse interacts with a first short low-pressure He gas cell, and the generated harmonics together with the remaining of the IR pulse successively interact with additional $\mathrm{He}$ targets. We will show numerically that by using such a

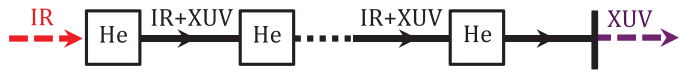

FIG. 3 (color online). Simplified scheme of the proposed experimental setup. configuration the generated radiation accumulates coherently and amplifies the XUV yield in the cutoff spectral region. In order to simulate the enhancement by iterative addition of the XUV pulses to the IR driving laser, we numerically filter the harmonics obtained from a first interaction of the IR pulse alone with a super-Gaussian of order 6 . The IR laser pulse and medium that we have considered have the same characteristics as in the previous study, shown in Figs. 1 and 2, so that the HHG cutoff is at $\approx 127 \mathrm{eV}$. The filtered harmonics should then be added to the IR pulse with a particular delay, and the resulting IR + XUV pulse should be sent again to interact with the medium, following an iterative procedure. However, as predicted by the results in Fig. 1, there is an XUV peak intensity threshold above which the recursive interaction with the gas target should produce the harmonics yield to grow for all delays except those where the IR field amplitude is null. In other words, as commented above, there is a value of the parameter $\alpha$ below which there is no need to fine-tune the delay between the XUV and the IR pulses, since basically all delays will produce XUV gain [see Figs. 1(c) and 1(d)]. Although in the present study we have not performed a detailed study of the threshold characteristics in general, we have observed the XUV gain threshold. Indeed, in order to have an XUV pulse combine with the IR pulse with a peak intensity above threshold, a sufficiently broad HHG spectrum needs to be filtered. We have thus considered the region $20-140 \mathrm{eV}$ covering all the high-order harmonics spectrum. As expected, this broad bandwidth provides an XUV peak intensity above threshold. In practice, this means we do not consider any filter in the generated harmonics and, as it will be shown below, we find that a zero delay in all iterations produces XUV amplification.

Figure 4 shows the results of the iterative procedure. As commented above, the filtered region of the spectrum is centered at $80 \mathrm{eV}$ and has a bandwidth of $120 \mathrm{eV}$. The delay is set to zero in each iteration, which in the present simulations means that the XUV pulse is added to the IR pulse delayed as it comes out from the output of the interaction. From Fig. 4 we observe that the amplification of the yield becomes progressively centered at around $125 \mathrm{eV}$, and thus at a much higher photon energy than the one corresponding to the center of the added XUV pulse. The enhanced spectrum is roughly $50 \mathrm{eV}$ wide at the 100th iteration. This can be understood by the fact that the spectral HHG region slightly above the cutoff has a lower XUV threshold, since in this region the harmonics yield is very low, and therefore the scattered spectral components in the cutoff region initially dominate the amplification. Specifically, the squared peak amplitude of the filtered XUV pulse is $E_{H}^{2}=8.2 \times 10^{-10}$ a.u., while the squared field amplitude of a Gaussian IR field with an intensity such as $5 \times 10^{14} \mathrm{~W} / \mathrm{cm}^{2}$ is $E_{0}^{2}=1.42 \times 10^{-2}$ a.u., which gives $\alpha=1.7 \times 10^{7}$. From the obtained results we can 


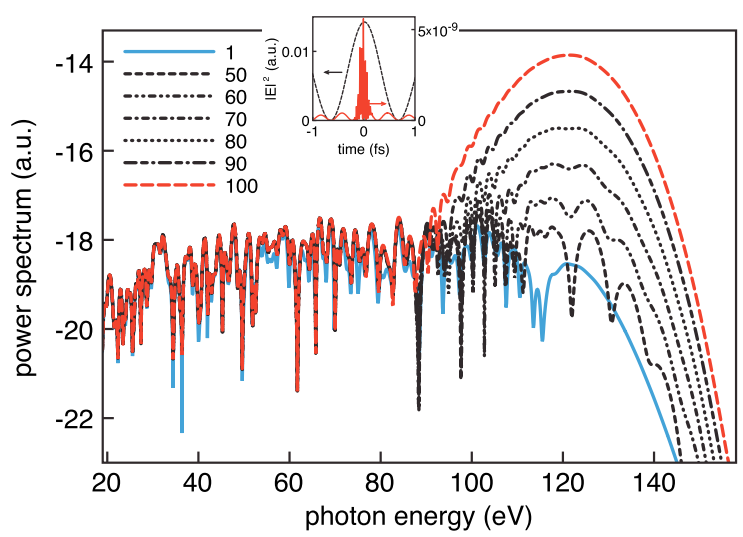

FIG. 4 (color online). XUV amplification obtained by filtering the harmonics spectrum at $80 \mathrm{eV}$ with $120 \mathrm{eV}$ spectral bandwidth. The filtered XUV is combined with the IR pulse as delayed as it comes out from the output of the interaction, in all iterations. The different lines correspond to the different iteration number, as indicated. The inset shows the filtered XUV pulse from the 100th iteration (red full line) compared with the input IR field (black dotted line). The vertical axis is the squared spectral field amplitude in atomic units and is given in logarithmic scale.

hence conclude that the XUV peak intensity is above threshold in the cutoff region, while it remains below threshold for the photon energies that are in the HHG plateau, which are not amplified. We estimate that the harmonics in the cutoff can be amplified by some 4 orders of magnitude at the 100th iteration, which corresponds to the generated radiation in that region adding coherently to the scattered XUV field. By using this consideration we calibrate the HHG conversion efficiency to $\approx 3 \times 10^{-7}$ in the present simulations. The inset in Fig. 4 shows how the XUV pulse, as filtered from the 100th iteration, becomes centered at zero delay. This is also expected since, although the harmonics from HHG are emitted at different times, their contributions near the center of the IR pulse become strongly amplified.

Further iterations will further amplify the XUV radiation. In order to account for XUV absorption in $\mathrm{He}$, we have repeated the previous simulations by reducing the energy of the XUV radiation at each iteration. The transmission in $\mathrm{He}$ at 30 torr, considering a propagation of $1 \mathrm{~cm}$, is $\approx 80 \%$ around $125 \mathrm{eV}$ [7]. For the low-pressure gas that we are considering, the losses by absorption are hence very small. In Fig. 5 we have considered XUV absorption losses of $10 \%$ and $15 \%$. We find that with $10 \%$ of losses, a higher amplification as that without losses is obtained at the 300th iteration, while 1000 iterations are needed in the case of $15 \%$.

In conclusion, we observe that XUV forward scattering from the nonstationary electronic wave packet promoted

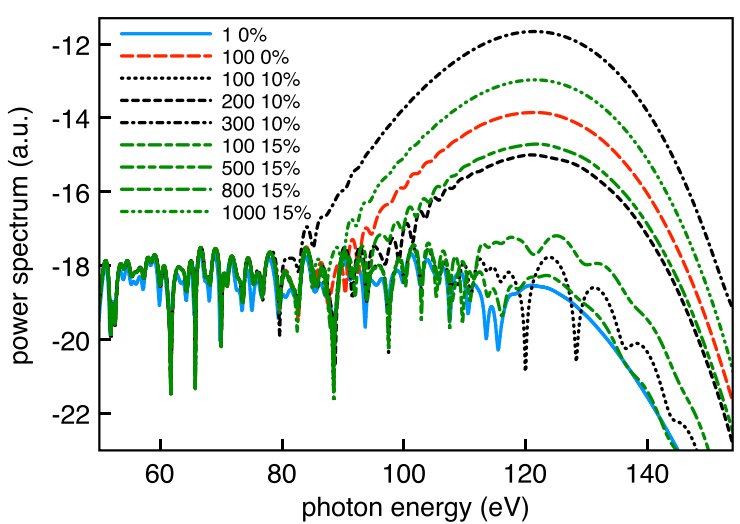

FIG. 5 (color online). Simulations as in Fig. 4 considering losses of $10 \%$ and $15 \%$, as indicated.

by an intense IR driving field in HHG processes dominates as compared with the normal weak scattering from bound or stationary free electrons. We predict that this effect can be used to amplify the HHG yield in the cutoff region, which sets the basis for a new coherent $\mathrm{x}$-ray source. The simulations that we have performed correspond to a $1 \mathrm{D}$ propagation of an intense IR field in $\mathrm{He}$ by neglecting phase-matching effects. This configuration can be approximated by the propagation of a barely focused strong IR pulse in a He gas cell with sufficiently low pressure for dispersion effects to be negligible. A more quantitative study is needed for a particular experiment, which might include other interaction geometries and also consider a different amplifying medium.

I am grateful to Vladislav S. Yakovlev for helpful discussions. Support from the Spanish Ministry of Economy and Competitiveness through "Plan Nacional" (FIS201130465-C02-02) is acknowledged.

*carles.serrat-jurado@upc.edu

[1] P. Agostini and L. F. DiMauro, Rep. Prog. Phys. 67, 813 (2004).

[2] K. Ishikawa, Phys. Rev. Lett. 91, 043002 (2003).

[3] K. J. Schafer, M. B. Gaarde, A. Heinrich, J. Biegert, and U. Keller, Phys. Rev. Lett. 92, 023003 (2004).

[4] C. Serrat, Appl. Sci. 2, 816 (2012); C. Serrat, Phys. Rev. A 87, 013825 (2013).

[5] M. Lewenstein, P. Balcou, M. Y. Ivanov, A. L'Huillier, and P. B. Corkum, Phys. Rev. A 49, 2117 (1994).

[6] See, e.g., J. Seres, E. Seres, D. Hochhaus, B. Ecker, D. Zimmer, V. Bagnoud, T. Kuehl, and C. Spielmann, Nat. Phys. 6, 455 (2010), and references therein.

[7] B. L. Henke, E. M. Gullikson, and J. C. Davis, At. Data Nucl. Data Tables 54, 181 (1993). 